\title{
Improving Analytical Spatial Resolution with the JEOL Field Emission Electron Microprobe
}

\author{
Peter McSwiggen ${ }^{*}$, Norihisa Mori**, Masaru Takakura*, ${ }^{* *}$ and Charles Nielsen ${ }^{* * *}$ \\ *McSwiggen \& Associates, 2855 Anthony Lane, St. Anthony, MN 55418; PMcS@McSwiggen.com \\ ** JEOL Ltd., 1-2 Musashino, 3 Akishima, Tokyo 196-8558 JAPAN \\ *** JEOL USA, Inc., 11 Dearborn Road Peabody MA 01960
}

Changes in column design and the electronics, as well as new types of spectrometers and analyzing crystals, have significantly advanced electron microprobes, in terms of stability, reproducibility and detection limits. Now a major advance in spatial resolution has come about through the use of the field emission electron gun. The spatial resolution of an analysis is controlled by the diameter of the electron beam and the amount of scatter that takes place within the sample. The smallest beam diameter is achieved at the highest accelerating voltages. However at these voltages, the scattering within the sample is at its greatest (Fig.1A). With a field emission electron gun, small beam diameters can be maintained even at very low accelerating voltages. As a result, the electron microprobe can work at accelerating voltages that produce only minimal scatter within the sample, producing a much smaller analytical area than either a thermionic tungsten or a $\mathrm{LaB}_{6}$ gun (Fig 2B). However there still is a trade-off between beam diameter and interaction volume, and as a result, the smallest analytical area is typically achieved at around $5-7 \mathrm{kV}$. At lower accelerating voltages, the increase in the beam diameter begins to overshadow the reduction in internal scattering.

The actual numerical size of the analytical area depends on the accelerating voltage, average atomic number, and the statistical definition of the size of the interaction volume within the target area. The number of X-rays generated within the sample decreases exponentially with distance from the beam, but there is no simple boundary that defines the limit of X-ray generation. Therefore some X-rays will be generated a great distance from the beam, but the probability becomes very small. As a result, in order to put a number on the size of the interaction volume, one must first define what percentage of the X-rays must be generated within that volume. When mapping, it is reasonable to accept a definition of the interaction volume whereby only $50 \%$ of the $\mathrm{X}$ rays must be generated within the target area. This will define with sufficient accuracy the phase boundaries. Using this definition, for a material like a Ag-Sn solder and using an accelerating voltage of $6 \mathrm{kV}$, the analytical area will be about $0.1 \mathrm{~mm}$. For quantitative analyses a more stringent definition of the interaction volume must be required. A much higher percentage of the X-rays must come from the target area for the analyses to be meaningful. For quantitative analyses, the analytical area would be considered to be $0.2 \mathrm{~mm}$ assuming $90 \%$ of the X-rays are required to come within the target area. This might be a suitable definition for analyses involving the major and minor elements. However if trace elements are involved, an even more stringent definition of the interaction volume would be needed, and in such a case, the analytical area would have to be considered to be in the order of $0.3 \mathrm{~mm}$.

The theoretical model for the analytical area was compared to actual results of measuring $\mathrm{Ag}_{3} \mathrm{Sn}_{1}$ particles of various sizes in a Sn matrix (Fig. 2). The measured k-ratios increase with increasing particle size, plateauing at the minimum particle size that can encompass analytical volume. At $6 \mathrm{kV}$, the plateau in the k-ratios occurs at about particle size of approximately $0.2-0.25$ microns in diameter. This agrees well with the theoretical calculations. 

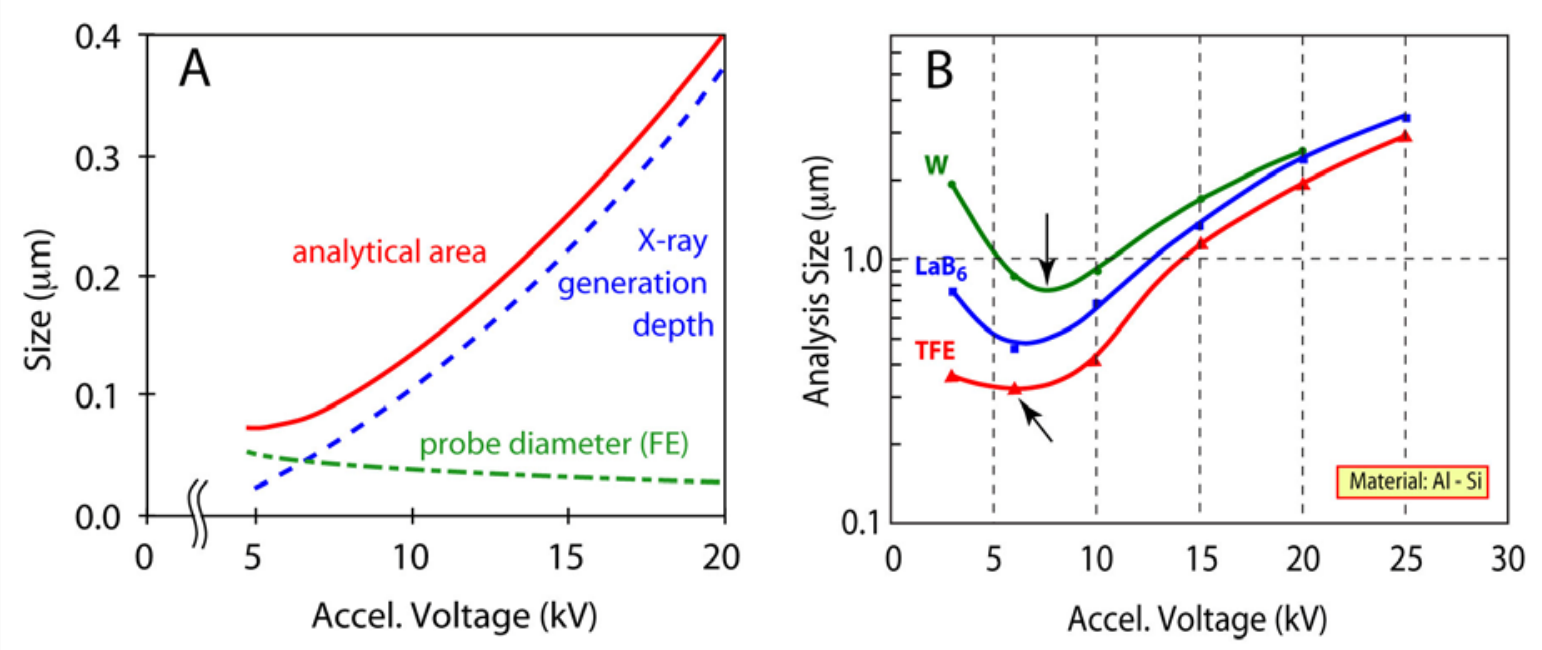

Figure 1. (A) Analytical area of a thermal-field emission gun (TFE) as a function of accelerating voltage. With decreasing accelerating voltage, the depth from which the X-rays are generated decreases, but the diameter of the beam increases. Therefore the analytical area reaches a minimum at around 5-7kV. (B) Comparison of the analytical area of a tungsten, $\mathrm{LaB}_{6}$, and TFE gun.

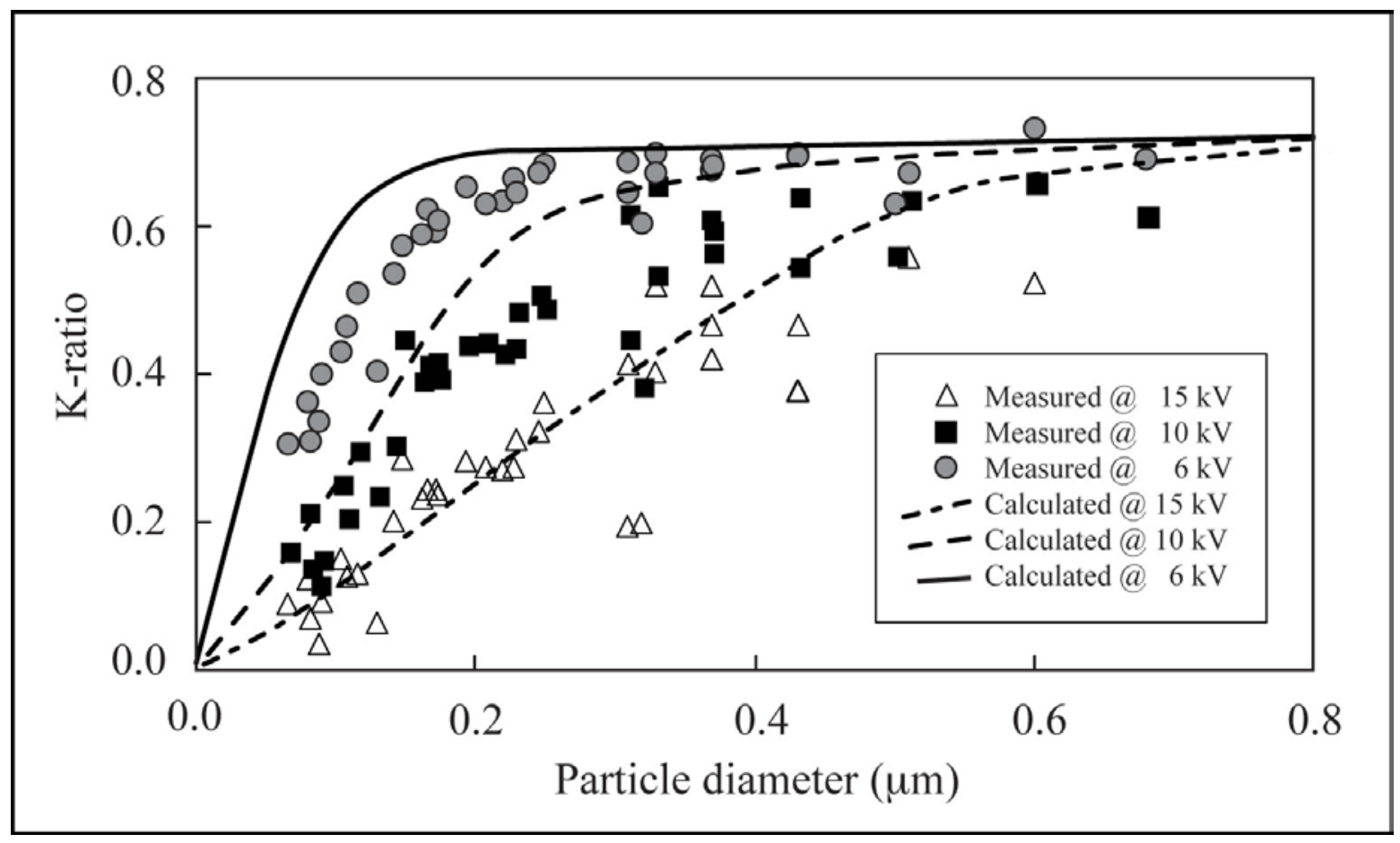

Figure 2. Measured Ag k-ratios from $\mathrm{Ag}_{3} \mathrm{Sn}_{1}$ particles in a tin matrix as compared to the theoretical calculations. As the particles increase in diameter, the k-ratio eventually reaches a plateau. This occurs at the minimum particle size that can encompass analytical volume. 